\title{
Quantum noise of a Michelson-Sagnac interferometer with a translucent mechanical oscillator
}

\author{
Kazuhiro Yamamoto, ${ }^{1,{ }^{*}}$ Daniel Friedrich, ${ }^{1}$ Tobias Westphal, ${ }^{1}$ Stefan Goßler, ${ }^{1}$ Karsten Danzmann, ${ }^{1}$ Kentaro Somiya, ${ }^{2}$ \\ Stefan L. Danilishin, ${ }^{3}$ and Roman Schnabel ${ }^{1}$ \\ ${ }^{1}$ Max-Planck-Institut für Gravitationsphysik (Albert-Einstein-Institut) and Institut für Gravitationsphysik, Leibniz Universität Hannover, \\ Callinstrasse 38, D-30167 Hannover, Germany \\ ${ }^{2}$ Theoretical Astrophysics, California Institute of Technology, Pasadena, California 91125, USA \\ ${ }^{3}$ Department of Physics, Moscow State University, Moscow RU-119992, Russia
}

(Received 16 December 2009; published 29 March 2010)

\begin{abstract}
Quantum fluctuations in the radiation pressure of light can excite stochastic motions of mechanical oscillators thereby realizing a linear quantum opto-mechanical coupling. When performing a precise measurement of the position of an oscillator, this coupling results in quantum radiation pressure noise. Up to now this effect has not been observed yet. Generally speaking, the strength of radiation pressure noise increases when the effective mass of the oscillator is decreased or when the power of the reflected light is increased. Recently, extremely light $\mathrm{SiN}$ membranes $(\approx 100 \mathrm{ng})$ with high mechanical $Q$ values at room temperature $\left(\geqslant 10^{6}\right)$ have attracted attention as low thermal noise mechanical oscillators. However, the power reflectance of these membranes is much lower than unity $(<0.4$ at a wavelength of $1064 \mathrm{~nm}$ ) which makes the use of advanced interferometer recycling techniques to amplify the radiation pressure noise in a standard Michelson interferometer inefficient. Here, we propose and theoretically analyze a Michelson-Sagnac interferometer that includes the membrane as a common end mirror for the Michelson interferometer part. In this topology, both power and signal recycling can be used even if the reflectance of the membrane is much lower than unity. In particular, signal recycling is a useful tool because it does not involve a power increase at the membrane. We derive the formulas for the quantum radiation pressure noise and the shot noise of an oscillator position measurement and compare them with theoretical models of the thermal noise of a SiN membrane with a fundamental resonant frequency of $75 \mathrm{kHz}$ and an effective mass of $125 \mathrm{ng}$. We find that quantum radiation pressure noise should be observable with a power of $1 \mathrm{~W}$ at the central beam splitter of the interferometer and a membrane temperature of $1 \mathrm{~K}$.
\end{abstract}

DOI: 10.1103/PhysRevA.81.033849

PACS number(s): 42.60.Da, 42.50.Lc, 07.60.Ly, 04.80.Nn

\section{INTRODUCTION}

Laser interferometers are among the most sensitive measurement devices ever built. The interferometric gravitational wave detectors in their first generation achieve a linear noise spectral density for the displacement measurement of as low as $10^{-19} \mathrm{~m} / \mathrm{Hz}^{1 / 2}$ [1]. The gravitational wave detectors of the second generation [2-4] are designed to have 10 times better sensitivity. The sensitivity of these interferometers will be limited by quantum radiation pressure noise [5] at low audio-band Fourier frequencies and by photon shot noise [5] at higher frequencies. While the shot-noise-limited regime of laser interferometers has been fully investigated and even interferometers with squeezed shot noise were demonstrated [6-9], the radiation pressure noise has not yet been observed. The experimental investigation of this quantum measurement regime is crucial in view of future gravitational wave detectors. It is also interesting from the fundamental physics point of view, because the successful observation of the quantum radiation pressure noise will confirm the seminal principle of back-action in a (continuous) quantum measurement [10,11] when one quantum system (the light) serves as a probe for another quantum system (the mechanical oscillator).

To observe quantum radiation pressure noise, the mechanical oscillator under investigation should have a high susceptibility to radiation pressure, i.e., a low effective mass [12]. The

*kazuhiro.yamamoto@aei.mpg.de motion of the oscillator should show a low thermal noise, i.e., the oscillator should have a high mechanical $Q$ value. Recently, commercially available SiN membranes have attracted a lot of attention and have been considered for experiments aiming for nonlinear quantum effects of mechanical oscillators, i.e., the observation of quantum jumps [13-15]. The membranes typically have an effective mass of the order of $100 \mathrm{ng}$, a thickness of about $100 \mathrm{~nm}$, and a surface area of about $1 \mathrm{~mm}^{2}$. The $Q$ values at their fundamental resonant frequency at about $100 \mathrm{kHz}$ were measured to $10^{6}$ at room temperature and $10^{7}$ at $300 \mathrm{mK}$ [14]. These properties of the membranes make them also interesting for experiments aiming for the linear quantum regime of light-matter systems as considered here. In such experiments, the dynamic (small) displacement of the oscillator scales linearly with the amplitude of the incident-light amplitude modulation, and the amplitude of the reflected-light phase modulation scales linearly with the (small) displacement of the oscillator. In an actual experiment, two identical oscillators might be used as the end mirrors of a simple Michelson interferometer with homodyne readout. A problem arises due to the rather low power reflectance of the membrane ( $<40 \%$ at a wavelength of $1064 \mathrm{~nm}$ ), because the interferometer techniques of power recycling and signal recycling [16-18] to amplify the radiation pressure noise are not efficient for translucent mechanical oscillators. Power recycling is used to resonantly enhance the light power inside the interferometer without reducing the signal bandwidth of the interferometer. Signal recycling is used to resonantly enhance the signal without increasing the laser power inside the interferometer. 


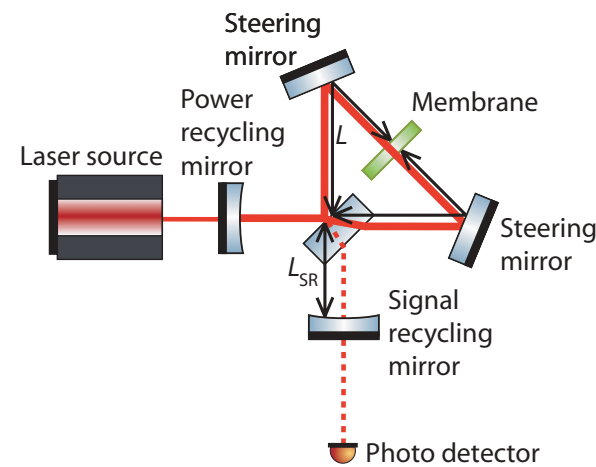

FIG. 1. (Color online) Schematic of a power- and signal-recycling Michelson-Sagnac interferometer. The translucent mechanical oscillator forms the joint end mirror of the two Michelson arms. While the displacement of the oscillator is measured via the Michelson mode, the transmitted light is stored in the Sagnac mode. The combination of Michelson and Sagnac modes enables the resonant enhancement of both the laser power and the displacement signal by placing power- and signal-recycling mirrors in the input and output ports of the interferometer, respectively. The parameter $L$ is the length of the Michelson interferometer arms, and $L_{\mathrm{SR}}$ is the distance between the beam splitter and the signal-recycling mirror.

Here we propose a recycling Michelson-Sagnac interferometer in order to access the radiation pressure noise regime of a translucent mechanical oscillator. In this interferometer topology, a single oscillator forms the joint end mirror of a Michelson interferometer with folded arms [19]. While the reflected light forms a Michelson mode, the light transmitted through the oscillator forms a Sagnac mode (see Fig. 1). Since all the light, including the signal, is kept within the two modes, power as well as signal recycling can be used to enhance the radiation pressure noise. We calculate the quantum noise of the Michelson-Sagnac interferometer and discuss the role of the power- and signal-recycling techniques for the observation of quantum radiation pressure noise. We finally compare the power spectral densities of radiation pressure noise, shot noise, and thermal noise for an oscillator displacement measurement and answer the question as to what values of light power and temperature of the membrane would allow an observation of radiation pressure noise.

\section{QUANTUM NOISE OF A MICHELSON-SAGNAC INTERFEROMETER}

For a displacement measurement of a mechanical oscillator, photon shot noise as well as radiation pressure noise has to be taken into account. In a Michelson-Sagnac interferometer, the corresponding spectral densities differ from those in a simple Michelson or Sagnac [20-22] interferometer due to the interference of the Michelson and the Sagnac modes.

\section{A. Photon shot noise of membrane displacement measurement}

Let us first consider a bare laser Sagnac interferometer that does not rotate. If the beam splitter has a perfect 50/50 splitting ratio, all the incident light is back-reflected toward the laser source. It is assumed that the Sagnac mode has a waist at half the round-trip length. In this case, a reflecting plane surface with amplitude reflectance $r$ (a membrane) can be put into the waist in such a way that the light is not scattered out of the interferometer and additionally creates a Michelson mode that is sensitive to the motion of the membrane. Note that the length change in each Michelson arm is twice the displacement of the membrane, and the differential length change in the two arms is twice times that change. Then the power at the output port is given by

$$
P_{\text {out }}=\frac{r^{2} P_{0}}{2}\left[1-\cos \left(\Phi_{0}+\frac{8 \pi}{\lambda} x\right)\right],
$$

where $x$ is the displacement of the membrane from its operating point $\Phi_{0}$, and $P_{0}$ is the incident power. If the displacement is much smaller than the wavelength of light $\lambda$, Eq. (1) can be approximated using a Taylor expansion yielding

$$
P_{\text {out }} \sim \frac{r^{2} P_{0}}{2}\left(1-\cos \Phi_{0}+\frac{8 \pi}{\lambda} x \sin \Phi_{0}\right) .
$$

The single-sided linear spectral density $\sqrt{G_{\text {out }}}$ of the shot noise of the light at the output port is described as [23]

$$
\sqrt{G_{\text {out }}}=\sqrt{2 \hbar \omega_{0} P_{\text {out }}}=\sqrt{\frac{4 \pi \hbar c P_{\text {out }}}{\lambda}},
$$

where $\hbar$ is the reduced Planck constant, $\omega_{0}(=2 \pi c / \lambda)$ is the angular frequency of light, and $c$ is the speed of light. The signal-normalized shot noise is then given by

$$
\begin{aligned}
\sqrt{G_{\text {shot }}} & =\sqrt{\frac{4 \pi \hbar c P_{\text {out }}}{\lambda}}\left[\left|\frac{\partial P_{\text {out }}}{\partial x}\right|_{x=0}\right]^{-1} \\
& =\sqrt{\frac{2 \pi \hbar c r^{2} P_{0}\left(1-\cos \Phi_{0}\right)}{\lambda}} \frac{2}{r^{2} P_{0}} \frac{\lambda}{8 \pi\left|\sin \Phi_{0}\right|} \\
& =\sqrt{\frac{\hbar c \lambda}{16 \pi r^{2} P_{0}}} \frac{1}{\left|\cos \left(\Phi_{0} / 2\right)\right|} .
\end{aligned}
$$

Hence, for $\Phi_{0}=0$ corresponding to the dark fringe, the signal-normalized shot noise is minimum for a given power $P_{0}$ :

$$
\sqrt{G_{\text {shot }}}=\sqrt{\frac{\hbar c \lambda}{16 \pi r^{2} P_{0}}} .
$$

\section{B. Quantum radiation pressure noise of membrane displacement measurement}

The radiation pressure force is equal to the momentum transferred from the light to the membrane per unit time. This is the difference of the light-field momentum flux between outgoing and incident beam, at the reflecting surface integrated over the effective area $\mathcal{A}$ of the light beam. The total momentum flux results from two pairs of incident $\left(E_{\mathrm{A}}, E_{\mathrm{B}}\right)$ and outgoing $\left(E_{\mathrm{C}}, E_{\mathrm{D}}\right)$ traveling waves (see Fig. 2). The momentum density for each wave is equal to $\epsilon_{0} \overline{\left|E_{\alpha}\right|^{2}} / c$, where $\alpha=\mathrm{A}, \mathrm{B}, \mathrm{C}, \mathrm{D}$. $E_{\alpha}$ is the electric-field strain in the corresponding wave, $\epsilon_{0}$ is the permittivity of vacuum, and $\overline{\left|E_{\alpha}\right|^{2}}$ stands for time average of $\left|E_{\alpha}\right|^{2}$ over many periods of light oscillation. Taking into 


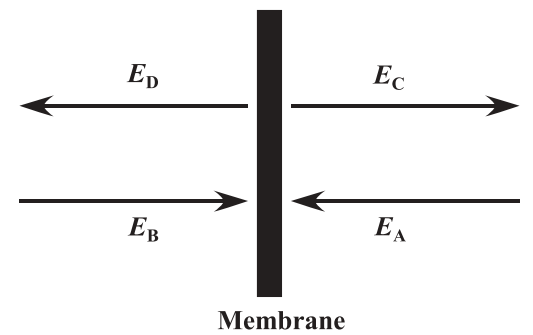

FIG. 2. The four arrows represent the incident-light fields $\left(E_{\mathrm{A}}, E_{\mathrm{B}}\right)$ and outgoing interference of the reflected and transmitted light $\left(E_{\mathrm{C}}, E_{\mathrm{D}}\right)$. These four fields give rise to radiation pressure effects at the membrane.

account the directions of light propagation and choosing signs in front of each wave momentum flux correspondingly, one gets the following expression for the radiation pressure force exerted by light on the membrane:

$$
F_{\mathrm{RP}}=c \times \mathcal{A} \frac{\epsilon_{0}}{c}\left[\left(\overline{\left|E_{\mathrm{C}}\right|^{2}}-\overline{\left|E_{\mathrm{D}}\right|^{2}}\right)-\left(\overline{\left|E_{\mathrm{B}}\right|^{2}}-\overline{\left|E_{\mathrm{A}}\right|^{2}}\right)\right] .
$$

The dc components of the force in Eq. (6) cancel because of the symmetry of the membrane. However, vacuum fluctuations enter the interferometer from the output port. This results in quantum amplitude fluctuations via the interference with the carrier light. In this case, there is a perfectly negative correlation between the fluctuations of $E_{\mathrm{A}}$ and $E_{\mathrm{B}}$ because of the energy conservation at the beam splitter [5]. This is the origin of quantum radiation pressure noise. The vacuum fluctuations from the input port do not lead to a displacement of the membrane, because the correlation between $E_{\mathrm{A}}$ and $E_{\mathrm{B}}$ is perfectly positive.

The incident-light-field amplitudes are

$$
\begin{aligned}
& E_{\mathrm{A}}=\frac{1}{\sqrt{2}} \sqrt{\frac{\hbar \omega_{0}}{\mathcal{A} c \epsilon_{0}}}\left[\sqrt{2} \mathcal{D}+\mathcal{E}_{\mathrm{v} 1}\right] \cos \left(\omega_{0} t\right) \\
& +\frac{1}{\sqrt{2}} \sqrt{\frac{\hbar \omega_{0}}{\mathcal{A} c \epsilon_{0}}} \mathcal{E}_{\mathrm{v} 2} \sin \left(\omega_{0} t\right), \\
& E_{\mathrm{B}}=\frac{1}{\sqrt{2}} \sqrt{\frac{\hbar \omega_{0}}{\mathcal{A c \epsilon _ { 0 }}}}\left[\sqrt{2} \mathcal{D}-\mathcal{E}_{\mathrm{v} 1}\right] \cos \left(\omega_{0} t\right) \\
& -\frac{1}{\sqrt{2}} \sqrt{\frac{\hbar \omega_{0}}{\mathcal{A c \epsilon _ { 0 }}}} \mathcal{E}_{\mathrm{v} 2} \sin \left(\omega_{0} t\right) .
\end{aligned}
$$

(In Ref. [24], these formulas are written in cgs-Gauss units. However, in this paper, we use International System of units.) Here $\mathcal{D}$ represents the amplitude of the carrier before the beam splitter, in such a way that $\mathcal{D}^{2}$ is the number of photons per unit time in the beam. The relation between $\mathcal{D}$ and the incident power $P_{0}$ is given by

$$
P_{0}=\hbar \omega_{0} \mathcal{D}^{2}
$$

The parameters $\mathcal{E}_{\mathrm{v} 1}$ and $\mathcal{E}_{\mathrm{v} 2}$ stand for amplitude and phase quadrature of vacuum fields, respectively, that enter the interferometer from the output port and propagate to the membrane. The phase shift of transmittance of the membrane is not independent of that of the reflectance owing to energy conservation $\left(\overline{E_{\mathrm{A}}^{2}}+\overline{E_{\mathrm{B}}^{2}}=\overline{E_{\mathrm{C}}^{2}}+\overline{E_{\mathrm{D}}^{2}}\right)$. Since the membrane is symmetric, the phase difference between the reflectance and transmittance must be $\pi / 2$. It is possible to assume a phase shift in the reflection of $\pi / 2$, while the phase shift in transmission is zero, without losing generality. The outgoing fields are written as

$$
\begin{aligned}
E_{\mathrm{C}}= & \frac{1}{\sqrt{2}} \sqrt{\frac{\hbar \omega_{0}}{\mathcal{A c \epsilon _ { 0 }}}}\left[\sqrt{2} t \mathcal{D}-t \mathcal{E}_{\mathrm{v} 1}+r \mathcal{E}_{\mathrm{v} 2}\right] \cos \left(\omega_{0} t\right) \\
& +\frac{1}{\sqrt{2}} \sqrt{\frac{\hbar \omega_{0}}{\mathcal{A c \epsilon _ { 0 }}}}\left[-\sqrt{2} r \mathcal{D}-r \mathcal{E}_{\mathrm{v} 1}-t \mathcal{E}_{\mathrm{v} 2}\right] \sin \left(\omega_{0} t\right), \\
E_{\mathrm{D}}= & \frac{1}{\sqrt{2}} \sqrt{\frac{\hbar \omega_{0}}{\mathcal{A c \epsilon} \epsilon_{0}}}\left[\sqrt{2} t \mathcal{D}+t \mathcal{E}_{\mathrm{v} 1}-r \mathcal{E}_{\mathrm{v} 2}\right] \cos \left(\omega_{0} t\right) \\
& +\frac{1}{\sqrt{2}} \sqrt{\frac{\hbar \omega_{0}}{\mathcal{A c \epsilon _ { 0 }}}}\left[-\sqrt{2} r \mathcal{D}+r \mathcal{E}_{\mathrm{v} 1}+t \mathcal{E}_{\mathrm{v} 2}\right] \sin \left(\omega_{0} t\right) .
\end{aligned}
$$

The parameters $r$ and $t$ are amplitude reflectance and transmittance of the membrane, respectively.

Substituting Eqs. (7), (8), (10), and (11) in Eq. (6), we obtain the force exerted by the radiation on the membrane

$$
F_{\mathrm{RP}}=\frac{2}{c} \sqrt{2 \hbar \omega_{0} P_{0}} r^{2} \mathcal{E}_{\mathrm{v} 1}+\frac{2}{c} \sqrt{2 \hbar \omega_{0} P_{0}} r t \mathcal{E}_{\mathrm{v} 2} .
$$

Let us assume that the field entering from the output port is a vacuum state. The single-sided spectral densities of $\mathcal{E}_{\mathrm{v} 1}$ and $\mathcal{E}_{\mathrm{v} 2}$ are equal to 1 (this spectral density has no dimension because the dimension of $\mathcal{E}_{\mathrm{v} 1}$ and $\mathcal{E}_{\mathrm{v} 2}$ are the same as that of $\mathcal{D}$, which is the square root of photon number per unit time) while their cross-correlation vanishes [24], thus one can easily get the following expression for single-sided spectral density of radiation pressure force

$$
\begin{aligned}
\sqrt{G_{F_{\mathrm{RP}}}} & =\sqrt{\left(\frac{2}{c} \sqrt{2 \hbar \omega_{0} P_{0}} r^{2}\right)^{2}+\left(\frac{2}{c} \sqrt{2 \hbar \omega_{0} P_{0}} r t\right)^{2}} \\
& =\sqrt{\frac{16 \pi \hbar r^{2} P_{0}}{c \lambda}} .
\end{aligned}
$$

The motion of the membrane caused by this force is

$$
\begin{gathered}
\sqrt{G_{\mathrm{rad}}}=H \sqrt{\frac{16 \pi \hbar r^{2} P_{0}}{c \lambda}}, \\
H=\left|\frac{1}{-m_{\mathrm{mem}}(2 \pi f)^{2}+m_{\mathrm{mem}}\left(2 \pi f_{\mathrm{mem}}\right)^{2}\left[1+\mathrm{i} f /\left(Q f_{\mathrm{mem}}\right)\right]}\right| .
\end{gathered}
$$

The function $H$ shows the (complex) mechanical susceptibility of the membrane, defined by its effective mass $m_{\text {mem }}$ [25], resonant frequency $f_{\text {mem }}$, and mechanical $Q$ value $Q_{\text {mem }}$.

\section{Power- and signal-recycling techniques}

The membrane in the Michelson-Sagnac interferometer can be positioned such that not only the Sagnac mode but also the Michelson mode is on a dark fringe at the signal port. In this case (almost) all the input light power is back-reflected toward the laser source and power and signal recycling [16-18] 
can be used to increase the quantum radiation pressure noise. Power- and signal-recycling techniques are realized via additional mirrors (see Fig. 1) which together with the Michelson-Sagnac interferometer form cavities for carrier light (power recycling) and signals and vacuum fluctuation entering the signal port (signal recycling). In this paper, we consider only the signal-recycling cavity tuned to the carrier frequency [26].

In the case of power recycling [16,17], the recycling cavity enhances the incident (carrier) power by a factor $g_{P R}$, which is the power-recycling (energy) gain. The incident power $P_{0}$ in formulas of shot noise [Eq. (5)] and radiation pressure noise [Eq. (14)] is replaced by $g_{\mathrm{PR}} P_{0}$. In the case of using signal recycling [18], the amplitude of sidebands caused by the membrane motion $\left[\left|\partial P_{\text {out }} / \partial x\right|\right.$ in Eq. (4)] and the vacuum fluctuations from the output port are amplified by $\sqrt{g_{\mathrm{SR}}}$, which is the signal-recycling (amplitude) gain.

It should be noted that the sideband frequency of the signal and the corresponding vacuum fluctuation is preferred to be smaller than the signal-recycling cavity bandwidth $f_{\mathrm{SR}}$. If the sideband frequency is larger than $f_{\mathrm{SR}}$, amplification of signal sidebands and vacuum fluctuation decreases. Hence, the signal-normalized shot noise increases, while the radiation pressure noise decreases. If the lengths of the two optical paths from the beam splitter to membrane are equal, the cutoff frequency $f_{\mathrm{SR}}$ is inversely proportional to the summation of the distance between beam splitter and signal-recycling mirror $L_{\mathrm{SR}}$ and the arm length $L$ (see Fig. 1). The formulas of shot noise and radiation pressure noise with power and signal recycling are written as $[18,27,28]$

$$
\begin{gathered}
\sqrt{G_{\mathrm{shot}}}=\sqrt{\frac{\hbar c \lambda}{16 \pi g_{\mathrm{PR}} g_{\mathrm{SR}} r^{2} P_{0}}} \sqrt{1+\left(\frac{f}{f_{\mathrm{SR}}}\right)^{2}}, \\
\sqrt{G_{\mathrm{rad}}}=H \sqrt{\frac{16 \pi \hbar g_{\mathrm{PR}} g_{\mathrm{SR}} r^{2} P_{0}}{c \lambda}} \frac{1}{\sqrt{1+\left(f / f_{\mathrm{SR}}\right)^{2}}} .
\end{gathered}
$$

If the reflectance of the recycling mirrors is close to unity but still lower than the reflectance of the Michelson-Sagnac interferometer, the cutoff frequency and recycling gains are

$$
\begin{gathered}
f_{\mathrm{SR}}=\frac{c\left(1-r_{\mathrm{SR}}\right)}{4 \pi\left(L_{\mathrm{SR}}+L\right)}, \\
g_{\mathrm{PR}}=\frac{1+r_{\mathrm{PR}}}{1-r_{\mathrm{PR}}}, \\
g_{\mathrm{SR}}=\frac{1+r_{\mathrm{SR}}}{1-r_{\mathrm{SR}}},
\end{gathered}
$$

where $r_{\mathrm{PR}}$ and $r_{\mathrm{SR}}$ are amplitude reflectance of power- and signal-recycling mirrors, respectively.

\section{SPECIFICATIONS FOR A LINEAR QUANTUM OPTO-MECHANICAL COUPLING}

In this section, we specify a design example of a MichelsonSagnac interferometer that should allow for the generation and observation of the linear quantum opto-mechanical coupling of a light field with the motion of a $\mathrm{SiN}$ membrane. The design parameters are chosen such that the regime dominated by radiation pressure noise can be reached for some low temperature of the membrane. In our analysis, we in particular
TABLE I. Example specifications of a Michelson-Sagnac interferometer [14].

Light wavelength $(\lambda)$

Arm length of Michelson interferometer $(L)$

Length between signal-recycling mirror and beam splitter $\left(L_{\mathrm{SR}}\right)$

Amplitude reflectance of signal-recycling mirror $\left(r_{\mathrm{SR}}\right)$

Signal-recycling (amplitude) gain $\left(\sqrt{g_{\mathrm{SR}}}\right)$

Power at beam splitter $\left(g_{\mathrm{PR}} P_{0}\right)$ (with/without signal recycling)

Power reflectance of membrane $\left(r^{2}\right)$

Resonant frequency of membrane $\left(f_{\text {mem }}\right)$

Effective mass of membrane $\left(m_{\text {mem }}\right)$

$Q$ value of membrane $\left(Q_{\text {mem }}\right)$

Temperature of membrane $\left(T_{\mathrm{mem}}\right)$

$1064 \mathrm{~nm}$

$0.6 \mathrm{~m}$

$3 \mathrm{~cm}$

0.998

32

$1 \mathrm{~W} / 1 \mathrm{~kW}$

0.35

$75 \mathrm{kHz}$

$125 \mathrm{ng}$

$10^{7}$

$1 \mathrm{~K}$

compared designs with and without signal recycling and found that the implementation of the signal-recycling technique is beneficial for the purpose of observing radiation pressure noise. The results of our comparison are based on the parameters given in Table I [14] and are illustrated in Fig. 3. In our design example, the radiation pressure noise is two times larger than the shot noise below resonant frequency; and even the standard quantum limit $[10,11,24]$, where shot noise and radiation pressure noise are of equal size, can be reached. Indeed, as one can see from Eq. (17), the optical power that determines the level of radiation pressure noise as well as shot noise always enters the formulas in the combination: $g_{\mathrm{PR}} g_{\mathrm{SR}} P_{0} /\left[1+\left(f / f_{\mathrm{SR}}\right)^{2}\right]$. Therefore for frequencies below the signal-recycling cavity cutoff frequency $f_{\mathrm{SR}}(76 \mathrm{kHz}$ for our choice of parameters), one needs $g_{\mathrm{SR}}$ times lower optical power $g_{\mathrm{PR}} P_{0}$ at the beam splitter to provide the same level of radiation pressure noise as in the the case without signal recycling. In our example, just $1 \mathrm{~W}$ is required when the signal-recycling technique is adopted compared to $1 \mathrm{~kW}$ without signal recycling. [We need a high signal-recycling gain $\sqrt{g_{\mathrm{SR}}}=32$. According to Eq. (20), the loss in the Michelson-Sagnac interferometer must be smaller than $0.4 \%$, which is a realistic value [29]]. Signal recycling reduces the heat from the absorption in the membrane and beam splitter without sacrificing the radiation pressure noise. For frequencies $>f_{\mathrm{SR}}$, signal recycling is less effective, as shown in Fig. 3 and Sec. II C. The signal-recycling cutoff frequency should therefore be designed to be above the observation band where the measurement is performed.

The observation of a quantum opto-mechanical coupling requires a low enough thermal noise. The off-resonant spectrum of thermal noise of an oscillator is not precisely known. Here, we consider two different dissipation mechanisms, namely, viscous and structural damping [30]. The thermal noise formulas are

$$
G_{\text {thermal }}=|H(f)|^{2} \frac{4 k_{\mathrm{B}} T_{\mathrm{mem}} m_{\mathrm{mem}}\left(2 \pi f_{\mathrm{mem}}\right)}{Q_{\mathrm{mem}}}
$$



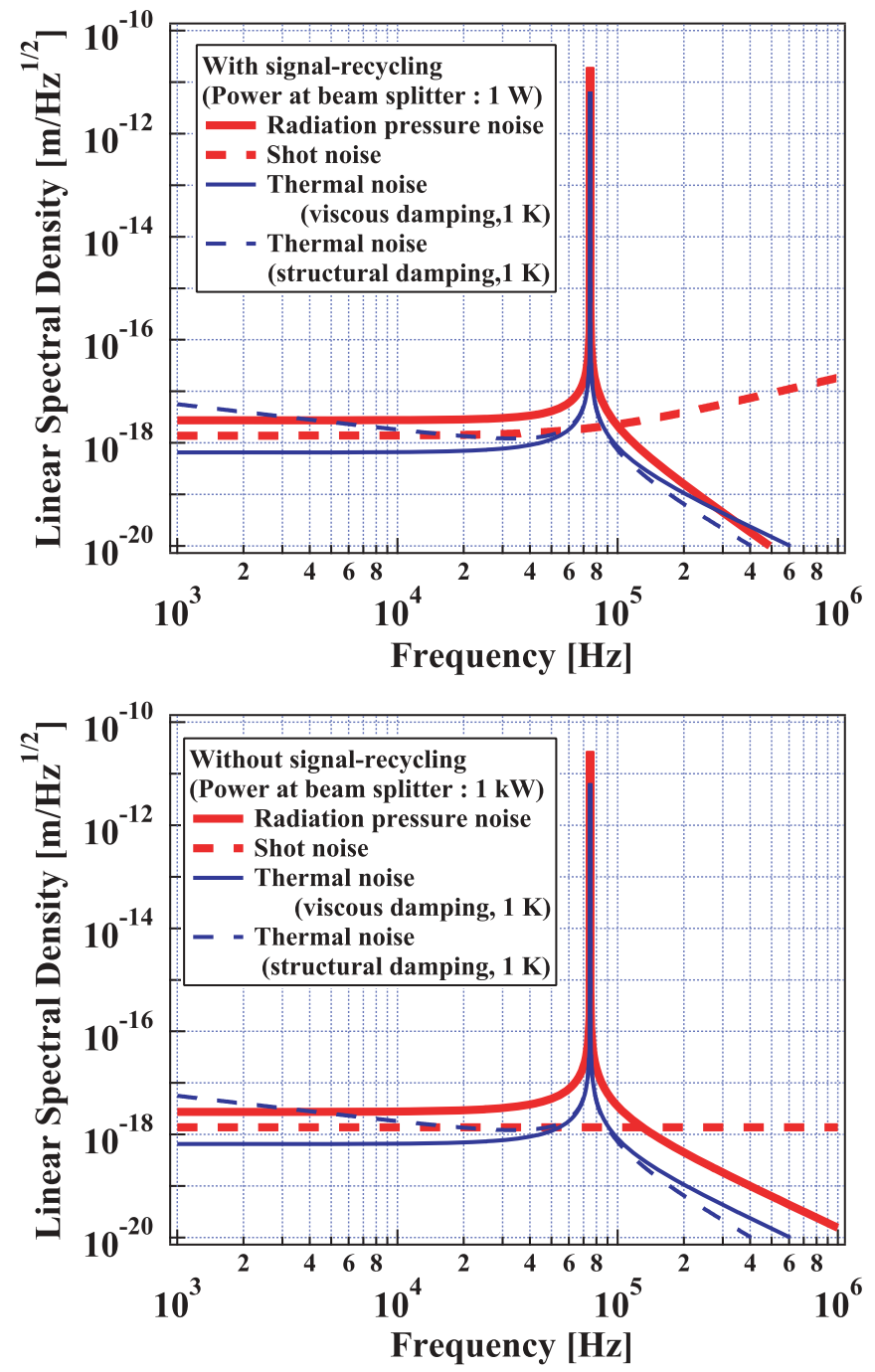

FIG. 3. (Color online) Goal sensitivity of the Michelson-Sagnac interferometer to measure radiation pressure noise based on the parameters in Table I. The graphs show the sensitivity with and without signal recycling, as labeled. Thick solid and dashed lines (red) are the radiation pressure noise and shot noise, respectively. Thin solid and dashed lines (blue) are thermal noise at $1 \mathrm{~K}$ in the cases of the viscous damping and structural damping [30].

$$
G_{\text {thermal }}=|H(f)|^{2} \frac{4 k_{\mathrm{B}} T_{\text {mem }} m_{\text {mem }}\left(2 \pi f_{\text {mem }}\right)^{2}}{Q_{\text {mem }}(2 \pi f)}
$$

(structural damping),

where $k_{\mathrm{B}}$ is the Boltzmann constant and $T_{\text {mem }}$ is the temperature of the membrane. Figure 3 shows the thermal noise of the membrane position measurement for the two dissipation mechanisms. For viscous damping the thermal noise spectrum is flat below the resonance (thin solid blue line). For structural damping the thermal noise spectrum falls with $1 / f^{1 / 2}$ (thin dashed blue line) because Fig. 3 shows the square root of Eqs. (21) and (22). Both thermal noise spectra are plotted for a membrane temperature of $1 \mathrm{~K}$. At this temperature, the thermal noise level is about a factor of 3 smaller than the radiation pressure noise around the membrane resonance frequency. At room temperature, the thermal noise is about 50 times larger, also partly because the $Q$ value of the membrane is 10 times smaller than at about $1 \mathrm{~K}$ [14]. In this case, the power at the beam splitter must be $3 \mathrm{~kW}$ even if signal recycling is adopted. Such a high power can in principle be achieved with the help of power recycling. However, light absorption in the membrane and in the interferometer will most likely cause thermal problems at these high powers. Note, that for a $Q$ measurement, a membrane temperature below $1 \mathrm{~K}$ was reached in Ref. [14].

To reduce the heat, the membrane should be placed at a node of the standing wave of the Sagnac mode. If the membrane is at the node, the output port is dark. The recycling techniques are compatible. Even at the node, the radiation pressure noise is not zero, because the radiation pressure noise depends on the momentum flux, not energy density as shown in Eq. (6). The signal sideband of the Michelson mode depends always linearly on the membrane displacement.

The displacement signal of the interferometer can be detected using a homodyne detector that is realized as a single photo diode if the Michelson-Sagnac interferometer is operated close to, but not exactly at, a dark fringe. In this case the interferometer laser field provides the optical local oscillator for homodyne detection. Alternatively, a balanced homodyne detector can be used if the interferometer is operated exactly at a dark fringe. Such a detection scheme uses a beam splitter, an external local oscillator field, and two photo diodes and was previously used as an interferometer readout in, for example, Refs. [8,9]. A balanced homodyne detector might be useful in order to increase the reflectance of the Michelson-Sagnac interferometer allowing for high signal-recycling gains.

\section{SUMMARY AND CONCLUSION}

Interferometer recycling techniques are useful in experiments that aim for the observation of radiation pressure noise of the position and displacement measurement of a mechanical oscillator. The Michelson-Sagnac interferometer topology, as proposed here, is compatible with power and signal recycling and is able to incorporate a mechanical oscillator that transmits a major part of the incident laser light. We have presented spectral densities for the shot noise and radiation pressure noise for a position measurement of a translucent $\mathrm{SiN}$ membrane. The expressions differ from those of a usual Michelson interferometer because of the interference between the beams reflected and transmitted by the membrane. Signal recycling can reduce the power in the interferometer required for pushing the radiation pressure noise above the shot noise. We have found that the radiation pressure noise of the interferometer signal is twice as large as the shot noise below the membrane resonant frequency of $75 \mathrm{kHz}$ for a laser power of $1 \mathrm{~W}$ incident on the beam splitter and a signal-recycling amplitude gain of 32 . To realize such a high signal-recycling gain, the Michelson-Sagnac interferometer has to show a high reflectance. Such a high value is realistic if the optical loss inside the interferometer is kept to a minimum (less than $0.4 \%$ ) and the interferometer is operated very close to a dark fringe. For an operation at exactly the dark fringe, a balanced homodyne detector has to be used. The membrane can be positioned in a node of the standing 
wave of the Sagnac mode in order to make absorption smaller without losing the proportionality between signal field and membrane displacement. If the membrane temperature is $1 \mathrm{~K}$, the calculated thermal noise of the oscillator fundamental mode is below the radiation pressure noise. This might turn out to be important in reducing the heating of the membrane due to absorbed laser power.

\section{ACKNOWLEDGMENTS}

This work is supported by the Deutsche Forschungsgemeinschaft and is part of Sonderforschungsbereich 407. K.S. is supported by the Japanese Society for the Promotion of Science. We are grateful to Yanbei Chen, Thomas Corbitt, and Albrecht Rüdiger for useful comments.
[1] S. E. Whitcomb, Class. Quantum Grav. 25, 114013 (2008).

[2] P. Fritschel, in Proceedings of the SPIE Meeting on Gravitational-Wave Detection (4856-39), Waikoloa, Hawaii, 2002, edited by M. Cruise and P. Saulson (SPIE, Bellingham, WA, 2003), p. 282.

[3] F. Acernese et al., J. Opt. A 10, 064009 (2008).

[4] K. Kuroda et al., Prog. Theor. Phys. Suppl. 163, 54 (2006).

[5] C. M. Caves, Phys. Rev. Lett. 45, 75 (1980).

[6] M. Xiao, L.-A. Wu, and H. J. Kimble, Phys. Rev. Lett. 59, 278 (1987).

[7] P. Grangier, R. E. Slusher, B. Yurke, and A. LaPorta, Phys. Rev. Lett. 59, 2153 (1987).

[8] K. McKenzie, D. A. Shaddock, D. E. McClelland, B. C. Buchler, and P. K. Lam, Phys. Rev. Lett. 88, 231102 (2002).

[9] H. Vahlbruch et al., Phys. Rev. Lett. 95, 211102 (2005).

[10] V. B. Braginsky and F. Ya. Khalili, Quantum Measurement, edited by K. S. Thorne (Cambridge University, Cambridge, UK, 1992).

[11] V. B. Braginsky and F. Ya. Khalili, Rev. Mod. Phys. 68, 1 (1996).

[12] T. J. Kippenberg and K. J. Vahala, Science 321, 1172 (2008).

[13] J. D. Thompson et al., Nature (London) 452, 72 (2008).

[14] B. M. Zwickl et al., Appl. Phys. Lett. 92, 103125 (2008).

[15] A. M. Jayich et al., New J. Phys. 10, 095008 (2008).

[16] R. W. P. Drever et al., in Quantum Optics, Experimental Gravity, and Measurement Theory, edited by P. Meystre and M. O. Scully (Plenum, New York, 1983), p. 503.

[17] R. W. P. Drever, in Gravitational Radiation, edited by N. Deruelle and T. Piran (North-Holland, Amsterdam, 1983), p. 321.
[18] B. J. Meers, Phys. Rev. D 38, 2317 (1988).

[19] T. Corbitt, Y. Chen, F. Khalili, D. Ottaway, S. Vyatchanin, S. Whitcomb, and N. Mavalvala, Phys. Rev. A 73, 023801 (2006).

[20] Y. Chen, Phys. Rev. D 67, 122004 (2003).

[21] S. L. Danilishin, Phys. Rev. D 69, 102003 (2004).

[22] Y. Chen, S. L. Danilishin, F. Ya. Khalili, and H. Müller-Ebhardt, QND measurements for future gravitational-wave detectors, e-print arXiv:0910.0319 [General Relativity and Gravitation (to be published)].

[23] W. A. Edelstein, J. Hough, J. R. Pugh, and W. Martin, J. Phys. E 11, 710 (1978).

[24] H. J. Kimble, Y. Levin, A. B. Matsko, K. S. Thorne, and S. P. Vyatchanin, Phys. Rev. D 65, 022002 (2001).

[25] K. Yamamoto, M. Ando, K. Kawabe, and K. Tsubono, Phys. Rev. D 75, 082002 (2007).

[26] Due to nonzero transmittance, the membrane modifies the optical path for both Sagnac and Michelson modes yielding into additional phase shift $\psi= \pm \arccos r$ between the ingoing and outgoing waves at the beam splitter. Therefore, the term "tuned recycling cavity" implies that the distance between the recycling mirror and the beam splitter should satisfy:

$$
L_{\mathrm{PR} / \mathrm{SR}}^{\mathrm{tuned}}=\frac{c}{\omega_{0}}\left(\pi N+\frac{\psi}{2}\right),
$$

with $N$ being integer.

[27] P. Fritschel, D. Shoemaker, and R. Weiss, Appl. Opt. 31, 1412 (1992).

[28] K. A. Strain and B. J. Meers, Phys. Rev. Lett. 66, 1391 (1991).

[29] D. Schnier et al., Phys. Lett. A 225, 210 (1997).

[30] P. R. Saulson, Phys. Rev. D 42, 2437 (1990). 